\title{
EXISTER DANS ET PAR LA CRISE. LA GESTION DE LA CRISE SANITAIRE COMME MODE DE LEGITIMATION DU « LOCAL »
}

\author{
Anne-Cécile DOUILLET \\ Professeure de science politique, université de Lille, CNRS, UMR 8026-Centre d'Études et \\ de Recherches administratives politiques et sociales (CERAPS)
}

\begin{abstract}
Résumé
À partir de l'étude de la presse nationale et de documents produits par les associations d'élus locaux et de fonctionnaires territoriaux, cet article analyse la façon dont les collectivités territoriales se sont données à voir entre mars et août 2020, via des discours et des politiques, et comment leur action et leurs prises de position ont pu être soutenues ou relayées par des éditorialistes, des journalistes, des experts. Ce faisant, l'article montre comment la «crise du coronavirus » a pu nourrir la cause décentralisatrice, par la publicisation de l'action des collectivités territoriales, mais aussi en fournissant de nouveaux arguments aux avocats de la décentralisation.
\end{abstract}

\section{Mots-clefs}

Collectivités locales, crise du coronavirus, décentralisation

\begin{abstract}
- Existing in and through the crisis. Management of the health crisis and legitimation of decentralisation - This article is based on a study of the national press and documents produced by associations of local elected representatives and territorial civil servants. It analyses first the way in which local and regional authorities presented themselves through speeches and policies between March and August 2020 and second how their actions and positions were supported or relayed by editorialists, journalists and experts. In doing so, the article shows how the "coronavirus crisis" was able to fuel the decentralising cause, by publicising the action of local authorities but also by providing new arguments to the advocates of decentralisation.
\end{abstract}

\section{Keywords}

Local governement, coronavirus crisis, decentralisation

Face à la crise sanitaire liée à la pandémie de Covid-19, en France, journalistes et analystes se sont interrogés sur le «retour de l'État» ${ }^{1}$, rendu plus «présent» par les interventions présidentielles répétées, mais aussi par la multiplication des mesures exceptionnelles, qu'il s'agisse de mesures restreignant les libertés ou de mesures de soutien aux

1 Antoine d'Abbundo et Marie Dancer, «Face au coronavirus, le retour de l'État», La Croix, 26 mars 2020 ; Philippe Escande, «Économie de marché : la crise du coronavirus provoque le grand retour de l'État », Le Monde, 5 avril 2020 
entreprises. Ce «retour de l'État» a été loué par certains, dénoncé par d'autres, suivant les effets craints $^{2}$ ou espérés ${ }^{3}$ de son intervention accrue. L'État dont il est question dans ces prises de position désigne de façon un peu vague « la puissance publique », mais plutôt telle qu'elle s'incarne dans les institutions du Gouvernement central, associées à l'État régalien ou à l'État providence. La crise sanitaire a cependant aussi été l'occasion d'un « retour des collectivités territoriales ». En effet, les collectivités territoriales ont non seulement multiplié les initiatives et les prises de parole, par la voix de leurs représentants élus notamment, mais le moment de crise est aussi apparu propice à la remise en cause d'une vision trop centralisée du Gouvernement et de l'action publique. Parmi les critiques, diverses, adressées à la gestion de crise par le Gouvernement français, celle d'une gestion trop «jacobine »s'est en effet fait entendre à plusieurs endroits ${ }^{4}$.

L'épisode de la crise sanitaire semble ainsi avoir constitué une fenêtre d'opportunité pour valoriser le rôle des élus locaux et défendre la décentralisation, peut-être d'autant plus facilement qu'il intervient après une période de relations nourries entre le Gouvernement et les élus locaux, qui ont aiguisé les enjeux de positionnement des derniers face au premier. Les premières années de la présidence Macron ont en effet été marquées par des épisodes de tension, sur les questions budgétaires en particulier ${ }^{5}$. La « crise des Gilets jaunes » de l'hiver 2018-2019 a ensuite favorisé une certaine revalorisation du local ${ }^{6}$, justifiant la mise à l'agenda du projet de

\footnotetext{
${ }^{2}$ Voir par exemple les analyses du politiste/historien Samuel Hayat : «La démocratie à l'épreuve du coronavirus », 23 mars 2020: https://samuelhayat.wordpress.com/2020/03/23/la-democratie-a-lepreuve-ducoronavirus/

${ }^{3}$ Gaël Giraud, « Covid-19 : le retour de l’État-providence ? », Libération, 25 mars 2020.

${ }^{4}$ Pierre-Yves Geoffard, « Covid-19 : les limites du jacobinisme sanitaire », Les Échos, 2 septembre 2020.

${ }^{5}$ Le « pacte girondin » proposé par Emmanuel Macron en juillet 2017 ne convainc pas les élus locaux, qui dénoncent les baisses de budget imposées aux collectivités territoriales et l'encadrement de la hausse des dépenses («pacte de Cahors»), y voyant une forme de recentralisation, malgré l'autonomie proclamée. La Conférence nationale des territoires de juillet 2018 est ainsi boycottée par les principales associations d'élus locaux.
}

${ }^{6} \mathrm{Ce}$ mouvement a en effet nourri une lecture en termes de retrait de l'État et de déconnexion entre le Gouvernement central et « les territoires ». Parmi de nombreux exemples d'analyses de ce type : Audrey Tonnelier, « Les "gilets jaunes" et la France "d'où la vie se retire” ", Le Monde, 14 janvier 2020. 
loi 3D (décentralisation, différenciation, déconcentration) $)^{7}$ : les concertations régionales qui commencent en janvier 2020 pour l'élaboration du projet de loi sont alors l'occasion pour les élus locaux d'exprimer un certain nombre de revendications. Dans ce contexte, l'irruption de l'épidémie et sa gestion gouvernementale ouvrent un nouvel espace de débat. Les collectivités territoriales ne se manifestent cependant pas uniquement par des prises de position critiques venant de leurs représentants. Elles sont aussi très mobilisées dans la gestion de la crise, mettant en œuvre une diversité de mesures visant à prendre en charge les conséquences du confinement, à organiser le déconfinement ou à prévenir la diffusion du virus. C'est donc et par le discours et par l'action que les collectivités territoriales se rendent visibles pendant les semaines de confinement et les mois qui suivent.

Par-delà une lecture stratégiste, qui chercherait à saisir la façon dont les représentants des collectivités territoriales se sont saisis de la crise pour renforcer leur légitimité, il s'agit ici d'appréhender plus largement la façon dont les collectivités territoriales se sont données à voir, via des discours et des politiques, à partir de la mi-mars 2020. L'enjeu de l'article n'est donc pas d'évaluer le poids « du local» dans la gestion de la crise ni de faire le bilan de la gestion locale de la crise sanitaire, mais plutôt d'analyser la façon dont les représentants des collectivités se sont manifestés pendant cette période, relayés par des éditorialistes, des journalistes, des universitaires...

L'analyse des initiatives prises par les collectivités territoriales, des prises de parole publiques les concernant et des controverses nouées autour de certaines décisions fait apparaître la « crise du coronavirus » comme un moment d'affirmation des collectivités territoriales et de leurs représentants. Entre mars et septembre 2020, les élus locaux ont multiplié les prises de parole, mais aussi les décisions, mettant en avant la pertinence de l'échelle infranationale pour gérer la crise. Même lorsque décision il y a, la symbolique l'emporte parfois sur le pouvoir d'orientation de l'action ou des comportements : la gestion de la crise apparait alors comme un vecteur de consolidation de la légitimité politique du local. Cependant, l'intervention des collectivités territoriales pendant la crise du coronavirus ne s'est pas limitée à une simple opération de communication et de mise en scène du pouvoir, même assortie de l'interpellation $\mathrm{du}$ Gouvernement central; il serait réducteur d'en rester à une analyse en termes

${ }^{7}$ Suite au « Grand débat national » organisé en réponse aux Gilets jaunes, le Président de la République annonce, le 25 avril 2019, sa volonté d'ouvrir « un nouvel acte de décentralisation adapté à chaque territoire ». 
d' « impuissance décisionnelle » (Le Bart, 2001). En effet, certaines interventions illustrent la capacité d'action des collectivités territoriales, parfois au-delà de leurs attributions, et alimentent des revendications d'extension des compétences des collectivités ou d'approfondissement de la décentralisation.

L'article s'appuie principalement sur une analyse de quatre titres de la presse nationale entre mars et août 2020 (Le Monde, Libération, Le Figaro, Les Échos). La presse est ici envisagée comme un espace public ouvert aux élus locaux, mais aussi aux représentants de l'État, à divers types d'experts, et dans le cadre duquel le travail journalistique façonne une certaine représentation des phénomènes sociaux ${ }^{8}$. Cet espace n'est évidemment pas également accessible à tous. C'est ainsi principalement aux « grands élus locaux » que la presse nationale donne la parole - aux maires de grande ville et présidents de région notamment, surtout s'ils ont (eu) des responsabilités nationales - même si des événements particuliers peuvent attirer l'attention sur d'autres élus. Si ce biais doit être pris en compte, il ne remet pas en cause le choix de la presse nationale pour étudier la parole des élus locaux : ces « grands élus » se posent en effet en représentants des collectivités territoriales et leur parole a une légitimité particulière, attachée à leurs responsabilités et/ou notoriété.

Cette recherche exploratoire sur les collectivités territoriales face à la crise sanitaire mériterait d'être complétée, notamment par des entretiens avec les élus les plus exposés dans la presse et les journalistes qui traitent les questions relatives aux collectivités territoriales, pour mieux comprendre les mécanismes de publicisation de l'action des collectivités territoriales et de leurs revendications. Elle pourrait aussi être prolongée par des enquêtes sur les mobilisations d'agents territoriaux et d'élus locaux visant à défendre la décentralisation dans ce contexte.

\section{LA CRISE COMME ESPACE D'EXPRESSION}

Analysant les attitudes des élus locaux des territoires touchés par la marée noire de l'Erika en 1999, C. Le Bart (2001) identifie trois postures: une posture décisionnelle, où

\footnotetext{
${ }^{8}$ Comme le rappelle Erik Neveu $(1999,45)$ en s'appuyant sur C. J. Calhoun, un espace public «n'est pas simplement, ni même premièrement, un espace de confrontation discursive d'arguments, mais aussi un site de propositions identitaires, de construction de communautés imaginées, de causes, de porte-parole. »
} 
l'action est centrale, même si elle peut s'accompagner de discours ; la posture performative, où le discours se confond avec l'action, comme lorsque, par exemple, les élus appellent à la mobilisation de la population ou interpellent les membres du Gouvernement; la posture expressive, dans les cas où il y a renonciation à l'action face à un constat d'impuissance, les élus occupant alors le terrain en exprimant les émotions censément partagées par la population (le découragement, la colère, etc.). Ces trois types de postures se retrouvent pendant la crise du coronavirus. Face à l'épidémie, les élus locaux ont en effet pris un certain nombre de décisions (voir infra), mais ils se sont aussi appuyés sur l'autorité associée à leur fonction pour exhorter la population à se protéger, dans une logique performative ${ }^{9}$, tout en exprimant parfois un sentiment d'impuissance ${ }^{10}$. Ce qui frappe surtout dans la façon dont nombre d'élus locaux ont mobilisé leur «droit de parole » (Le Bart, 1998) pendant la crise sanitaire de 2020, c'est que l'enjeu semble être de se montrer agissants face à leurs administrés, mais aussi de se positionner face à l'État central. À travers leurs prises de parole comme à travers les décisions qu'ils prennent, c'est à la fois comme détenteurs d'une légitimité à agir propre au « local » et comme incarnation d'un contre-pouvoir face au Gouvernement que les élus locaux se posent.

\section{La rhétorique bien huilée de la proximité et des défaillances de l'État}

Même si la valorisation de la proximité peut être le fait d'acteurs gouvernementaux lorsqu'ils défendent par exemple la justice ou la police de proximité - elle est particulièrement prisée des élus locaux, qui sont les principaux vecteurs de cette rhétorique. Ils font de la proximité un signe distinctif, en lien avec leur ancrage et leur connaissance du «terrain ». Comme le souligne R. Lefebvre (2005, 103): «ils ne cessent d'exalter la valeur

${ }^{9}$ Début mars 2020, avant les mesures de confinement prises par le Gouvernement, le maire de La Balmede-Sillingy (74), testé positif au virus, s’adresse ainsi aux habitants de sa commune, par vidéo : « Je vous en prie, ne faites plus la bise, ne serrez plus de mains et déplacez-vous seulement si vous en avez besoin ».

${ }^{10}$ Le Parisien du 26 mars 2020 rapporte ainsi les propos de Régis Pinot, maire d'une commune de la Haute-Saône, lui-même testé positif au Covid-19 : « Je suis très en colère, oui. Ce manque de matériel, en France, la cinquième puissance mondiale, c'est impensable! (...) Je ne comprends pas qu'un pays comme le nôtre se retrouve dans cette situation, ajoute-t-il. On avait des milliards de masques. » 
"irremplaçable" du terrain qui permet de "prendre le pouls de la population", "d'écouter ses doléances", de comprendre ses aspirations ", la proximité étant par ailleurs associée à "l'expérience » et au "pragmatisme ». Ces arguments n'ont pas manqué d'être mobilisés à l'occasion de la crise du coronavirus, notamment au moment du déconfinement, comme l'illustre cette intervention de M. Aubry dans la matinale de France Inter, le 30 avril 2020, à quelques jours du déconfinement prévu pour le 11 mai :

«Tout ce qui est difficile à faire est renvoyé aux maires. Je pense que c'est une bonne chose parce que nous avons la réalité du terrain et les réponses peuvent être différentes d'une commune à l'autre. »

La maire PS de Lille souligne ainsi la capacité des édiles à affronter les difficultés, du fait de l'avantage que procure la connaissance du terrain, qui permet des réponses adaptées et différenciées. Nombre d'élus locaux, et pas seulement municipaux, ont ainsi vanté une approche localisée de la crise :

«En donnant les clés du déconfinement aux maires, [Édouard Philippe] répond à la demande que nous ne cessons de formuler depuis le début de cette crise inédite : faire enfin confiance au local.» (A. Robinet, maire LR de Reims, Le Monde, 29 avril 2020)

«Tout est piloté depuis Paris, mais Paris n'est pas la France! Il y a un vrai besoin de décentralisation. On l'a vu avec les "gilets jaunes", on le voit encore avec les turbulences causées par le Covid. » (A. Rousset, président PS de la région Nouvelle-Aquitaine, Le Figaro, 23 avril 2020)

La défense du local s'accompagne souvent d'une critique des manques et manquements de l'État :

« Oui, je considère que je compense les défaillances de l'État, si les maires sont en première ligne, c'est parce qu'il n'y a pas de réponse nationale. » (P. Saurel, maire sans étiquette de Montpellier, Libération, 20 avril 2020)

«Partout, les collectivités doivent suppléer les défaillances de l'État. Cette crise met en évidence, encore une fois, l'impuissance de l'État et à quel point il est désarmé. » (S. Troussel, président PS du conseil départemental de la Seine-Saint-Denis, Le Monde, 24 avril 2020) 
Cette critique n'est pas univoque dans ses implications. Si le maire de Montpellier, qui fait ici référence à la commande de masques, se pose alors en recours face aux défaillances de l'État grâce à la réactivité ${ }^{11}$ et la capacité de contrôle du local (" J'en ai commandé un million, en activant les filières locales. On en distribuera deux par personne, en prenant garde à éviter les détournements. »), le président de la Seine-Saint-Denis regrette pour sa part la tendance de l'État à renvoyer ce qu'il ne parvient pas à faire vers les collectivités territoriales, tout comme la présidente de la région Occitanie, qui en conclut cependant qu'il faut donner plus de pouvoirs aux régions :

«Les collectivités ne peuvent pas continuer de tout anticiper et de tout prévoir à la place de l'État »(S. Troussel, Le Monde du 30 avril 2020)

«Ce n'était pas aux régions de faire ça, et surtout pas à elles de payer. Nous avons agi à la place de l'État! (...) C'est bien nous qui étions en première ligne! Nous ne sommes pas dans la rancune ou dans l'amertume, mais dans le constat qu'en France il faut donner plus de pouvoir aux régions. » (C. Delga, présidente PS de la région Occitanie, Le Figaro, 20 mai 2020)

Ce type de position s'inscrit en fait dans une logique critique plus large, où se mêlent critique de l'État, de la centralisation et du gouvernement en place.

\section{Le pouvoir d'interpellation : s'imposer comme force critique}

C'est parfois le Gouvernement et sa gestion qui sont directement visés. En interpellant et critiquant le Gouvernement, les élus locaux se constituent alors, de facto, en "force d'opposition ». Si la thématique de la proximité sous-tend une légitimation par la plus grande efficacité ou pertinence de l'action publique, cette logique d'opposition gouvernementale pose les élus locaux dans un rôle démocratique de contre-pouvoir, un rôle proprement politique, et pas seulement de gestionnaires des affaires publiques. Une telle posture a peut-être d'autant plus d'écho que, du fait de l'état d'urgence sanitaire, le rôle du Parlement est réduit et les

${ }^{11}$ Dans la défense des collectivités territoriales, la proximité est de plus en plus associée à la « réactivité » et à l'《 agilité ». 
parlementaires moins appelés à s'exprimer. Si les enjeux partisans ne sont pas complètement absents, c'est bien en tant qu'élus locaux qu'ils s'expriment et c'est cet attribut qu'ils mettent en avant.

Certaines prises de position sont d'ailleurs collectives, affichant les élus locaux - ou certaines catégories d'élus - comme un groupe d'opposition gouvernementale. Ainsi, le 3 mai 2020, 316 maires d'Île-de-France ${ }^{12}$ signent une lettre ouverte publiée dans La Tribune et relayée par Libération, Les Échos et Le Figaro ${ }^{13}$. Les élus s'adressent à Emmanuel Macron pour demander le report de l'ouverture des écoles après le 11 mai, jugeant la chose irréaliste. La démarche est certes liée à un domaine de compétence qui est le leur (les écoles maternelles et primaires), et les maires attirent l'attention sur leur responsabilité, refusant d'endosser les conséquences d'une politique qui n'est pas la leur; elle n'en fait pas moins des élus locaux une force critique mettant en cause la politique gouvernementale de déconfinement, d'autant que la presse relaie nombre d'exemples de maires s'exprimant dans le même sens à titre individuel ${ }^{14}$.

Les prises de position médiatiques des élus locaux entre mars et septembre 2020 ont été d'autant plus frappantes qu'elles ont parfois dépassé les champs d'intervention qui sont les leurs. Un épisode particulièrement illustratif est ici celui du débat sur la vente de masques par la grande distribution. En effet, cette commercialisation n'engage pas directement les compétences des collectivités territoriales ni les rapports État/collectivités. Ce n'est pas le Gouvernement qui est ici directement en cause, mais des acteurs économiques, dont l'activité est nationale et non locale. Pourtant, des élus locaux s'étonnent et s'offusquent publiquement de l'attitude des grandes enseignes. Début mai 2020, Renaud Muselier, président LR de la région Provence-Alpes-Côte d'Azur et président de Régions de France ${ }^{15}$, donne ainsi « trois jours à la grande distribution pour prouver qu'elle n'avait pas de stock secret de masques

${ }^{12}$ Après le ralliement d'autres signataires, le nombre total atteindra finalement 394.

13 « Les maires d'Île-de-France ne porteront pas la responsabilité de l'État dans une réouverture des écoles à marche forcée », La Tribune, 3 mai 2020.

${ }^{14}$ Voir par exemple : Nelly Didelot, « Les maires ont-ils le droit de s'opposer à l'ouverture des écoles ? », Libération, 2 mai 2020.

${ }^{15}$ Régions de France $(\mathrm{RdF})$ représente les régions et collectivités régionales de métropole et d'outre-mer. 
pendant la crise!", ajoutant : "Si cela se confirme, je déposerai immédiatement plainte au pénal. » (Le Figaro, 4 mai 2020.) Si cette menace est plus relayée dans la presse locale que dans la presse nationale ${ }^{16}$, elle pose néanmoins Renaud Muselier dans un rôle politique large, dépassant le cadre des politiques régionales.

\section{La mise en scène du pouvoir local et des contraintes étatiques}

Par-delà ces prises de parole publiques, la gestion de la crise sanitaire a aussi donné lieu à une multiplicité d'initiatives de la part des collectivités territoriales, dans le cadre de leurs compétences ou sur des fondements juridiques plus incertains. Certaines apparaissent assez largement symboliques, dans le sens où la dimension communicationnelle semble l'emporter sur les effets attendus des décisions. Il s'agit alors d'exister politiquement, en mettant en scène le pouvoir décisionnel, quelle que soit sa portée. Donner à voir une volonté d'action peut aussi s'accompagner d'une monstration des limites du pouvoir local, notamment dans une logique d'opposition à l'État : il s'agit alors de parer des critiques ou de dénoncer des contraintes légales ou financières - pour engager un rapport de force. Certaines initiatives locales ont de fait donné lieu à des luttes, sur le terrain communicationnel ou juridique, quant à leur légitimité ou leur légalité. Si l'issue de ces luttes a été variable, elles ont mis en débat la place des collectivités territoriales et la portée de la décentralisation.

Associant leur critique des défaillances de l'État à l'action, plusieurs élus locaux ont pris des mesures signifiant leur souci de prendre la lutte contre la propagation de l'épidémie à bras le corps. P. Laurent, maire UDI de Sceaux et secrétaire général de l'AMF ${ }^{17}$, adopte ainsi le 6 avril 2020 un arrêté rendant obligatoire le port du masque dans les rues de sa commune. Si cet arrêté est suspendu par la justice administrative, comme d'autres de même nature par la suite, il permet au maire à la fois d'afficher son souci de sécurisation de ses concitoyens, de

${ }^{16}$ Dans les quatre titres dépouillés, seul cet article du Figaro le rapporte, alors que l'épisode est commenté dans trois articles de La Provence et quatre de Nice Matin, entre le 2 et le 5 mai 2020. La « colère » de Renaud Muselier est cependant également relayée sur les réseaux sociaux et dans les médias audiovisuels nationaux.

\footnotetext{
${ }^{17}$ Association des maires de France et des présidents d'intercommunalité.
} 
dénoncer le défaut de protection par l'État ${ }^{18}$ et de faire de l'État un frein aux initiatives locales. En mars, l'adoption d'arrêtés de couvre-feu par certaines municipalités pour «durcir le confinement $»^{19}$ s'inscrit dans les mêmes mécanismes de démonstration d'autorité, d'autant plus dans ce cas que les sorties sans motif sont de toute façon interdites de jour comme de nuit. Même si la justice administrative ne tranche pas toujours dans le même sens (Donier, 2020), plusieurs de ces arrêtés sont suspendus et certains maires sont conscients qu'ils jouent avec la légalité :

« Je reconnais que quelquefois, sur certains sujets, je peux être à la limite de la légalité. Mais je n'allais pas laisser au bord du chemin des administrés, des entreprises, des gens en grande précarité, en grande situation de fragilité. » (C. Estrosi, maire LR de Nice, Le Figaro, 11 avril 2020)

Se retrouvent ici des modes d'agir déjà observés en d'autres circonstances, qui relèvent d'une forme de «communication médiatique par arrêtés municipaux » (Markus, 2020). De récents arrêtés «anti-pesticides » ou contre l'installation d'antennes-relais ont ainsi permis à certains maires de prendre position contre des politiques gouvernementales ; les arrêtés «antimendicité » ou de couvre-feu des années 1990 et 2000 avait déjà donné lieu à des batailles juridiques et médiatiques opposant certains maires à l'État. Comme l'analyse T. Le Goff(2005, 430), la réalité pratique de ces arrêtés est limitée, "d'autant plus qu'ils sont régulièrement annulés par le juge administratif », mais ils "possèdent en revanche une portée symbolique », car «ils mettent en scène la volonté d'un maire de maintenir l'ordre en exposant des signes ostensibles de son autorité », ce d'autant plus que les médias leur donnent une certaine visibilité. De manière plus anecdotique, les mesures prises ici ou là pour désinfecter les rues, retirer les bancs, limiter les distances de sortie ou les achats, interdire de cracher, mettre en place des barrages routiers relèvent de la même logique.

L'épisode de l'achat de masques par certaines collectivités peut être lu comme un autre exemple de démonstration d'autorité : il s'agit là aussi d'agir sur des bases légales incertaines

${ }^{18}$ Interrogé par le New York Times, le maire de Sceaux reproche à l'État de ne plus être « capable de répondre au besoin de sécurité » des Français (cité par Courrier international du 30 avril 2020 : Audrey Fisné, « Vu de l'étranger. La centralisation française fragilisée par la crise »).

${ }^{19}$ Le $1^{\mathrm{er}}$ avril, Le Monde recensait plus de 170 communes ayant pris ce type d'arrêté. 
pour donner priorité à l'action. Ces collectivités, dont certaines ont commandé des masques dès le mois de mars (pour leurs administrés, leurs agents ou les personnels soignants), ont d'abord été rappelées à l'ordre par l'État, qui a réquisitionné les masques commandés par certaines régions pour les personnels les plus exposés. Cependant, la mobilisation des élus locaux n'a pas été sans effet : un décret du 21 mars 2020 les autorise à faire leurs propres importations. Par ailleurs, si le ministère de la santé rappelle alors que ce sont les agences régionales de santé (ARS) et les préfets qui sont chargés de l'approvisionnement et de la distribution vers les professionnels de santé, il accepte que cela se fasse en coordination avec les collectivités territoriales. Enfin, les collectivités territoriales ont obtenu que les masques achetés à partir du 13 avril soient remboursés par l'État à hauteur de $50 \%$.

Ce dernier exemple montre que la posture des élus locaux n'a pas été que communicationnelle dans la gestion de la crise, ils ont aussi fait montre d'une capacité à agir. Si la crise leur a ouvert un espace de visibilité, elle les a aussi conduits à déployer un certain nombre de mesures. Si celles qui viennent d'être évoquées aboutissent à un bras de fer avec l'État, d'autres ont été déployées dans une logique moins conflictuelle, souvent dans le prolongement des missions assurées habituellement par les collectivités. La valorisation de ces actions a nourri la défense d'un approfondissement de la décentralisation.

\section{LA CRISE AU SERVICE D'UNE DEMANDE DE DECENTRALISATION}

En appelant la mise en œuvre d'actions spécifiques, la crise sanitaire a fourni des arguments aux avocats de la décentralisation. En effet, les politiques entreprises ont pu être présentées comme une preuve du bien-fondé de la décentralisation. Par ailleurs, certaines « expérimentations » amorcées en réponse à la crise ou certaines contraintes subies dans le déploiement d'action ont pu servir de fondement à de nouvelles propositions.

\section{La preuve par l'action}

Les collectivités territoriales ont été des actrices importantes de la gestion de la crise. Elles ont agi dans le cadre de leurs compétences et des services dont elles assurent la gestion, 
mais aussi, parfois, au-delà. Sans pouvoir étudier la réception de la gestion locale de la crise par les citoyens, nous analyserons la façon dont les élus, et parfois les fonctionnaires territoriaux, ont valorisé leur action et leurs initiatives dans la presse et comment celle-ci a pu servir de caisse de résonnance.

Il serait ici impossible de lister la diversité des actions entreprises par les collectivités territoriales en lien avec l'épidémie, dont la presse, locale et nationale, rend compte. Les collectivités communales et intercommunales ont notamment dû assurer la continuité d'un certain nombre de services, comme la collecte des déchets ou les transports urbains; les départements ont, pour leur part, dû réorganiser nombre de services, comme ceux de l'aide à l'enfance ; certaines collectivités ont par ailleurs déployé des moyens pour apporter un soutien aux personnes fragiles et isolées et/ou soutenu l'ouverture de centres de consultation dédiés au dépistage des personnes contaminées, sans compter la distribution de masques et différentes formes de soutien aux personnels soignants. Certaines initiatives sont l'occasion pour les collectivités de souligner, chiffres à l'appui, l'effort important qu'elles déploient dans ce contexte de crise ; d'autres sont l'occasion de rappeler le rôle qu'elles jouent, en continu ${ }^{20}$.

Fin avril/début mai, le rôle joué par les élus locaux, notamment les maires, « premiers de cordée du déconfinement $»^{21}$, est fortement mis en avant dans la presse ${ }^{22}$. Sans oublier leur rôle en matière éducative ou de transport, les régions ont particulièrement valorisé le rôle qu'elles ont joué dans le soutien aux entreprises, en déployant différentes aides mises en place dans le cadre de leurs compétences économiques, mais aussi en abondant le fonds national de solidarité pour le soutien aux entreprises ${ }^{23}$. Dans cette exposition de la mobilisation des collectivités

${ }^{20}$ Pour un exemple de présentation des actions entreprises par les collectivités territoriales, voir : « Régions et départements unis face au Covid », Le Monde, 2 avril 2020.

21 « Les maires labeur au ventre... », Libération, 28 avril 2020.

${ }^{22}$ Voir aussi, par exemple : « Les élus locaux sur le devant de la scène », Le Monde, 30 avril 2020.

${ }^{23}$ Plusieurs articles de presse rendent compte des actions mises en œuvre par les régions, soulignant souvent leur « réactivité ». Voir par exemple : « Face à la crise, les régions se mobilisent », Le Monde, 6 mai 2020 , ou encore « Les élus locaux en première ligne pour pallier les carences de l'État», Le Figaro, 21 avril 2020. 
territoriales, les départements apparaissent un peu moins visibles ${ }^{24}$, même si leurs représentants ont aussi mis en avant à quel point ils avaient été « en première ligne », « tout particulièrement sur le front sanitaire, social et médico-social », en faisant le bilan de leur action ${ }^{25}$. Certaines initiatives ont par ailleurs été l'occasion de montrer non seulement la volonté des collectivités départementales de prendre leur part dans la prise en charge des conséquences de la crise, mais aussi de leur capacité à engager des fonds pour cela. Certains départements se sont en effet engagés dans des politiques dépassant leurs compétences pour soutenir des entreprises en difficulté : ils ont créé leurs propres fonds de soutien ${ }^{26}$ - ce qui relève de la compétence des régions - ou abondé ceux des régions, alors que seul le bloc communal a cette faculté. Ces initiatives, qui ont parfois créé des tensions avec les régions, ont conduit à un rappel à l'ordre de l'État sur la répartition des compétences issue de la loi NOTRe de 2015, par la voix de la ministre en charge des Relations avec les collectivités territoriales. Elles rouvrent cependant le débat sur les compétences des conseils départementaux. Les actions déployées par les collectivités territoriales pendant la crise sanitaire ont en effet nourri de nouvelles propositions de décentralisation.

\section{Des arguments pour les avocats de la décentralisation}

Les élus locaux n'ont pas été les seuls défenseurs de l'action des collectivités territoriales dans ce contexte de crise sanitaire : des journalistes, des experts et d'autres acteurs engagés

\footnotetext{
${ }^{24}$ Un indice de cette moindre exposition est la plus faible présence médiatique du président (D. Bussereau) de l'Association des départements de France (ADF), qui contraste avec celle du président de Régions de France (R. Muselier), même si la différence est déjà notable avant la crise. Dans les 4 titres nationaux exploités ici, 106 articles mentionnent R. Muselier entre le $1^{\mathrm{er}}$ mars 2020 et le 31 août 2020, contre 76 dans les 6 mois précédents. D. Bussereau passe lui de 35 à 42 mentions.

${ }^{25} \mathrm{http}: / /$ www.departements.fr/mesures-mises-place-departements-cadre-de-letat-durgence-sanitaireliee-a-lepidemie-de-covid-19-2/ Voir aussi l'article cité en note 20 et « Les départements se mobilisent pour soutenir le secteur touristique », Le Monde, 14 mai 2020.

${ }^{26}$ Le département des Bouches-du-Rhône a ainsi mis en place un « fonds territorial et solidaire pour la sauvegarde de l'emploi ». Les Alpes-Maritimes ont aussi créé un « fond d'urgence » pour soutenir les entreprises du département.
} 
dans la vie des collectivités se sont aussi faits les avocats de la décentralisation, en «tirant les leçons de la crise ». C'est parfois un niveau territorial plutôt qu'un autre qui est défendu ${ }^{27}$, mais les initiatives communes des collectivités territoriales sous la bannière de Territoires unis ${ }^{28}$ ou d'associations de fonctionnaires territoriaux font aussi exister une demande de décentralisation venant des « territoires », par-delà les luttes institutionnelles.

Dans la presse, internationale comme nationale, diverses analyses ont attribué les « mauvaises performances » dans la gestion de crise à l'excessive centralisation française. Même s'il est peu probable que la "réactivité » ou les meilleurs résultats dans la gestion de crise s'expliquent uniquement par l'organisation territoriale - le cas allemand a souvent été mis en avant pour défendre la décentralisation, beaucoup moins les cas espagnol ou italien, pourtant caractérisés par une assez forte autonomie régionale -, celle-ci est mobilisée comme un argument éclairant les difficultés rencontrées en France. Cette critique se retrouve dans la presse internationale ${ }^{29}$, mais aussi dans la presse française. Le Figaro du 20 avril 2020 titre ainsi l'un de ses articles : « la centralisation de l'État a-t-elle ralenti le traitement de la crise ? ». Le 2 mai, un ancien journaliste publie une tribune dans Libération sur l'échec de la centralisation : «Le fiasco entourant la gestion du Covid-19 en France est paradoxalement porteur d'une bonne nouvelle: l'échec de la centralisation ${ }^{30}$. Le lien entre mauvaise gestion de la crise et centralisation est également fait par des chercheurs en sciences sociales : O. Borraz, directeur de recherche au CNRS, demande ainsi d' "en finir avec le monopole d'État sur les gestions de crise $»^{31}$. Pour R. Pasquier, également directeur de recherche au CNRS, "la défaillance

${ }^{27}$ Le niveau régional apparaît particulièrement défendu, mais le département trouve aussi des avocats (voir le plaidoyer et les propositions précises de X. Patier, fonctionnaire territorial, dans Le Figaro du 20 mai 2020 [«Les départements reprennent des couleurs »] et les propos du géographe L. Chalard, qui invite à rouvrir le dossier de la décentralisation en privilégiant l'échelon départemental, la gestion de la crise l'ayant d'après lui « remis à l'honneur » [voir notamment Les Échos, 13 mai 2020]).

${ }^{28}$ Qui regroupe les trois grandes associations d'élus représentant le bloc communal, les départements et les régions.

${ }^{29}$ Voir l'article de Courrier international cité en note 18.

${ }^{30}$ Voir aussi note 5.

${ }^{31}$ Titre donné à un entretien qu'il a donné dans Médiacités, paru le 22 avril 2020. 
systémique mise en lumière par cette crise résulte principalement d'une centralisation excessive et d'une trop grande rigidité des procédures. L'État doit se recentrer sur ses missions essentielles $»^{32}$.

Ce sont cependant les représentants des collectivités territoriales qui se manifestent le plus dans les appels à décentraliser dans le prolongement de la crise sanitaire - les élus locaux bien sûr, comme cela a déjà été dit $^{33}$, mais aussi d'autres acteurs territoriaux :

«Cela nous conforte dans l'idée qu'on défend : ce sont le bloc communal, les acteurs départementaux et régionaux qui sont en capacité d'organiser les services de proximité. » (E. Verlhac, directeur général de l'AMF, Libération, 21 avril 2020)

«Dans une logique de subsidiarité, les collectivités territoriales sont les mieux placées pour proposer des mesures locales de nature à garantir l'atteinte des objectifs gouvernementaux au moyen, le cas échéant, de mesures innovantes et différenciées, selon les caractéristiques locales. » $\left(\mathrm{AATF}^{34}, 2020,13\right)$

Les administrateurs territoriaux ne se sont d'ailleurs pas contentés de défendre l'approfondissent de la décentralisation comme ligne directrice, au nom de "l'agilité des collectivités territoriales »(AATF, 2020, 12), ils ont également formulé des propositions précises, à partir d'une enquête par questionnaire : dix propositions concluent leur rapport, concernant à la fois les relations entre les collectivités territoriales et l'État (pour une plus grande concertation, plus de subsidiarité) et les objectifs à encourager en matière d'action publique territoriale (la relocalisation des activités prioritaires, le développement durable, le numérique, la relance économique), mais aussi des dispositions plus précises permettant d'accroître les marges de manœuvre des autorités locales ("faciliter sous forme

${ }^{32}$ Figaro Vox, 5 mai 2020

33 Pour une autre illustration: «Face à la crise, Pécresse plaide pour un "grand mouvement de décentralisation” », Le Figaro, 22 juin 2020.

${ }^{34}$ Association des administrateurs territoriaux de France. Les administrateurs territoriaux sont les agents qui détiennent les grades les plus élevés de la filière administrative de la fonction publique territoriale. 
d'expérimentation l'entrée au capital de sociétés commerciales pour les EPCI $\left.{ }^{35}\right)$. Le rapport est par ailleurs émaillé de propositions en relation avec les différents thèmes abordés, comme la «possibilité d'activation d'une compétence économique d'exception en aides directes pour tous les échelons sur un temps de crise bien défini ou du moins systématiser le conventionnement entre les régions et les autres échelons ». Cette proposition répond aux initiatives, évoquées ci-dessus, de certains départements en matière économique.

Les associations d'élus se sont également mobilisées pendant ce temps de crise pour, collectivement, demander une remise à l'agenda de la décentralisation et une nouvelle répartition des compétences, en s'appuyant sur les « leçons de la crise ». Le 30 mai 2020, dans une tribune publiée dans $L e$ Monde $^{36}$, les présidents de région demandent ainsi au chef de l'État de faire «le pari des territoires », via « un plan de relance et de reconstruction coconstruit avec les régions », mais aussi en fixant « une grande ambition décentralisatrice à notre pays », laquelle passe notamment par le renforcement du pouvoir des conseils régionaux. Dans le cadre des discussions engagées dans le cadre du projet $3 \mathrm{D}$, les associations d'élus répètent qu'il faut tirer les conclusions de la gestion de la crise sanitaire, en accordant plus de pouvoirs aux collectivités territoriales. Tel est le discours porté par exemple par Territoires unis, qui met sur la table différentes propositions en juillet 2020 : faire passer une partie de Pôle emploi aux régions, faire des départements les pivots des politiques sociales et médico-sociales (avec notamment la responsabilité du pilotage des EHPAD). Les associations d'élus locaux vantent elles aussi « l'agilité des territoires », face aux « limites d'un État corseté par une centralisation bureaucratique et lointaine $»^{37}$.

35 Voir «"La solution à la crise passe par les territoires" Regards croisés des administrateurs et administratrices territoriaux sur le Covid-19» dans le présent dossier thématique.

\footnotetext{
36 «Pour la relance et la reconstruction, les régions sont prêtes !»

${ }^{37}$ Territoires unis, Plus de liberté pour plus d'efficacité, 8 juillet 2020, 20 p.
} 
Étudiant l'exercice des pouvoirs de police administrative pendant l'état d'urgence sanitaire, V. Donier (2020) conclut dans le présent dossier thématique à une «forte tendance recentralisatrice », une « décentralisation réduite à la portion congrue » et des autorités locales (les maires en l'occurrence) «posées en relais des mesures nationales ». Notre propos souligne pour sa part la place prise par les collectivités territoriales pendant la même période. Les deux conclusions ne sont cependant pas contradictoires, elles adoptent simplement des angles de vue très différents. En effet, notre étude s'intéresse moins à la décentralisation - en tant qu'état des places respectives de l'État et des collectivités territoriales - qu'à la cause décentralisatrice, en se plaçant non pas sur le terrain du droit, mais sur celui du débat public. Il apparaît alors que la cause décentralisatrice a pu bénéficier de la crise sanitaire. D’un côté, les élus locaux - les maires et les présidents d'exécutifs régionaux notamment - ont été assez présents dans l'espace médiatique : les initiatives locales ont ainsi été rendues visibles et les élus ont aussi joué un rôle de critique de la politique gouvernementale, se posant souvent en recours face aux défaillances de l'État. Les élus locaux se sont faits les chantres de la décentralisation en paroles et en actes. Par ailleurs, certaines initiatives en marge des compétences des collectivités territoriales ont reposé la question du contour de celles-ci, alimentant directement certaines propositions, qui trouvent à s'exprimer dans le cadre de la préparation de la loi 3D. Tout cela ne préjuge évidemment pas des évolutions à venir en matière de décentralisation ni de l'influence qu'auront les associations d'élus locaux ou de fonctionnaires territoriaux. L'analyse qui précède ne donne aucun élément permettant de l'apprécier, d'autant qu'il aurait été possible, de la même manière, d'exposer des prises de position contre la décentralisation ${ }^{38}$. Si elle ne fait que constater que le contexte de crise sanitaire a été propice aux constructions argumentatives en faveur de la décentralisation, cette recherche exploratoire contribue néanmoins à éclairer les dynamiques des réformes de décentralisation en France. En montrant comment les élus locaux,

${ }^{38}$ Voir par exemple : P. Steinmetz, « Nos ratés face au Covid-19 n’ont aucun rapport avec un manque de décentralisation », Le Figaro, 25 avril 2020 
par diverses voies, portent les arguments en facteur d'une plus grande décentralisation et se mobilisent à travers leurs associations, cet article tend finalement à conforter l'hypothèse du rôle "moteur et offensif» des élus locaux dans les processus de réforme du système d'administration français (Le Lidec, 2007).

\section{Références bibliographiques}

AATF (2020), Retour d'expérience sur la gestion de la crise sanitaire pour penser le monde d'après, rapport téléchargeable sur https://www.administrateursterritoriaux.asso.fr/contenu/uploads/2020/06/200626_AATF_Retour_Gestion_Crise_V 5.pdf

Donier, Virginie (2020), «Qui fait la police (administrative) sous l'état d'urgence sanitaire ? », Revue française d'administration publique, $\mathrm{n}^{\circ}$ 176, 2020/4.

Le Bart, Christian (1998), Le Discours politique, Paris, PUF.

Le Bart, Christian (2001), « Leadership et impuissance décisionnelle. Les élus locaux face à la marée noire de 1'Erika », Sciences de la société, nº 53, p. 25-41.

Le Goff, Tanguy (2005), "L'insécurité "saisie" par les maires. Un enjeu de politiques municipales », Revue française de science politique, vol. 55, n 3, p. 415-444.

Lefebvre, Rémi (2005), « La proximité à distance. Typologie des interactions élus-citoyens », in Lefebvre, Rémi et Le Bart, Christian, dir. (2005), La Proximité en politique, Rennes, PUR, p. 103-127.

Le Lidec, Patrick (2007), « Le jeu du compromis : l'État et les collectivités territoriales dans la décentralisation en France », Revue française d'administration publique, vol. 121-122, $\mathrm{n}^{\mathrm{o}} 1$, p. 111-130.

Markus, Jean-Paul (2020), «Communication médiatique par arrêtés municipaux », AJDA, p. 753.

Neveu, Erik (1999), « Médias, mouvements sociaux, espaces publics », Réseaux, vol. 17, n 98, p. 17-85. 